\title{
Cross-Sectional Imaging of Materials Structure Using PS-OCT
}

\author{
M.R. Stra̧kowski, J. Pluciński, And B.B. Kosmowski* \\ Department of Optoelectronics and Electronic Systems \\ Faculty of Electronics Telecommunications and Informatics \\ Gdańsk University of Technology, Narutowicza 11/12, 80-952 Gdańsk, Poland
}

\begin{abstract}
Optical low-coherence tomography is a measurement technique for non-contact and non-destructive investigation of materials inner structure. Nowadays, this method is highly applied in medical treatment especially in dermatology and ophthalmology. During our research we have developed an optical low-coherence tomography system with polarization state analysis for structure examination of a broad range of technical materials. In this paper we present our recent measurements including polarization sensitive analysis. Those measurements were taken for semitransparent and highly scattering materials like polymer layers and polymer optical retarders, anticorrosion protective coatings or abrasive paper. We proved that with the aid of developed polarization sensitive optical coherence tomography system it is possible to investigate birefringence principles of materials as well as to improve visualization contrast of cross-sectional images.
\end{abstract}

PACS numbers: 42.79.--e, 42.87.--d

\section{Introduction}

Optical low-coherence tomography (OCT) is one of the most rapidly advancing techniques. This method is capable for non-contact and non-destructive investigation of inner structure of a broad range of materials. OCT combines low-coherence interferometry and heterodyne detection with lateral point beam scanning to produce two- or three-dimensional images [1]. Nowadays, this method is highly applied in medical treatment especially in dermatology and ophthalmology and also in industry and science [2]. Recent researches on OCT expand their applications to materials characterization, surface and subsurface defect localization [3, 4], art conservations [5] and many other fields beyond medical applications. During our research we have been concentrated on polarization sensitive optical coherence tomography (PS-OCT). In contrast to conventional OCT, in which the

*corresponding author; e-mail: kosmos@eti.pg.gda.pl 
magnitude of received interference signals is recorded, PS-OCT can record the anisotropic structure in an investigated material [5]. Polarization sensitive analysis gives extra information about birefringence principles of investigated materials and also increase contrast ratio of OCT imaging, which can be useful, for example, to map strain fields in investigated device [3, 4]. The polarization sensitive analysis is based on polarization diversity detection. Therefore, it is possible to separate interference signals obtained for two orthogonal polarization states of backscattered light. All calculations are carried out using the Jones formalism, in which the polarization properties of the sample are described by the depth-resolved Jones matrix. According to the Jones formalism, a non-depolarizing non-dichroic material can be analyzed as an optical retarder. Therefore, the fast axis orientation and retardation angle provided by the sample completely describe the birefringence of the investigated materials [6-9]. A setup of developed PS-OCT system and its principles of operation have been described in our previous works [10, 11].

\section{Measurement results}

The polarization sensitive analysis gives unique benefits to OCT imaging, which was mentioned in Sect. 1. In order to assess the usefulness of this analysis for non-biological materials investigation a series of tests were carried out. Those tests included an investigation of backscattered light intensity, as well as local anisotropy changes. As a result, the cross-sectional images of surface and subsurface scattering centers and local optical retardation provided by investigated material were produced. For tests semitransparent as well as highly scattering samples were chosen. In this elaboration we present OCT images obtained for sand-paper with aluminium oxide grits, polycrystalline lead zirconium titanate (PLZT) ceramics, and polymer optical retarders. The results of the experiment are shown in Figs. 1 to 5. For our experiments the PS-OCT was used. This system was developed by the authors and its features have been summarized in Table.
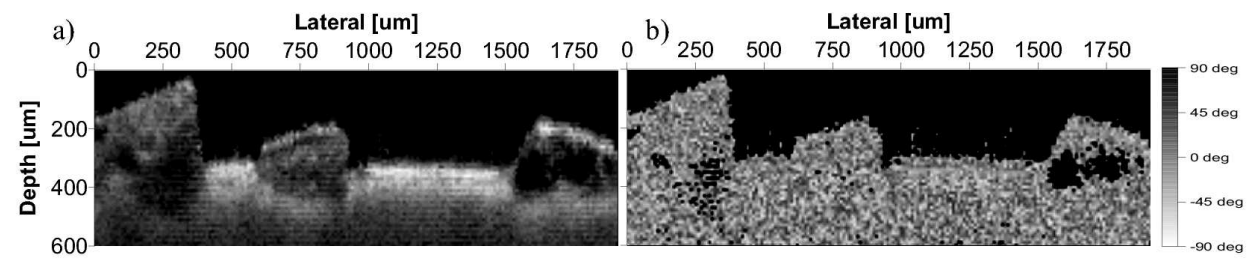

Fig. 1. OCT images of sand-paper: (a) intensity image, (b) optical retardation image.

Our studies have been focused on improving visualization contrast and quantitative determination of anisotropic changes in materials structure. The OCT images in Fig. 1 and Fig. 2 were taken for sand-paper and PLZT ceramic material. Comparing the intensity images (Fig. 1a and Fig. 2a) with optical retardation 


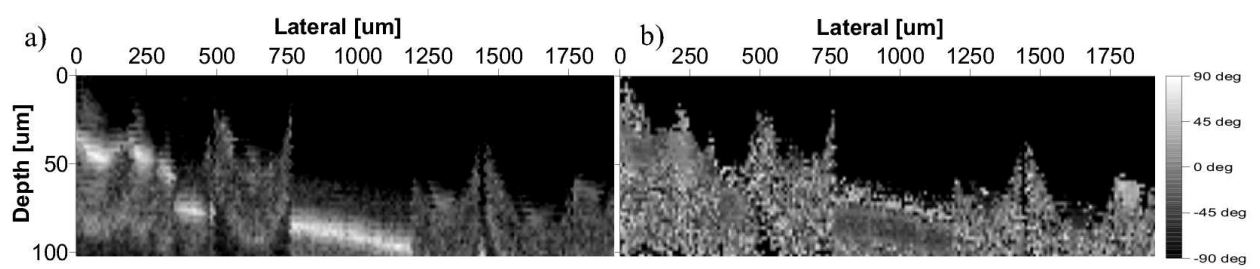

Fig. 2. OCT images of PLZT ceramic material: (a) intensity image, (b) optical retardation image.

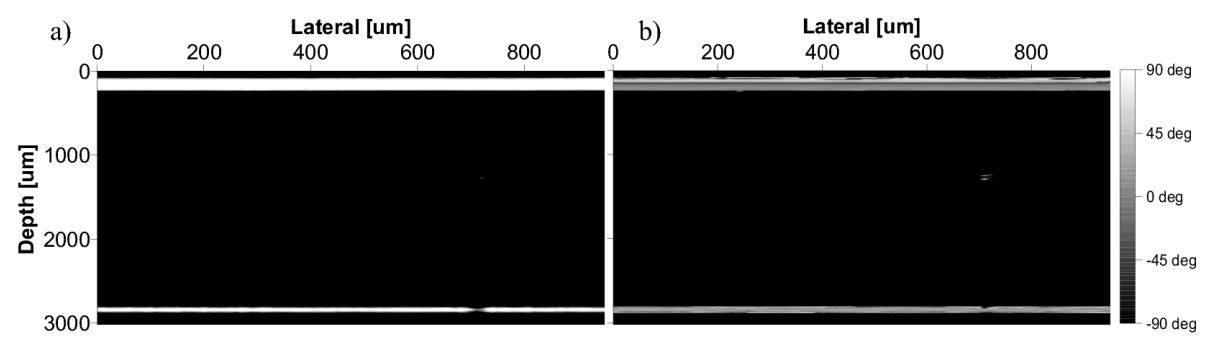

Fig. 3. OCT images of polymer retarder 1: (a) intensity image, (b) optical retardation image.

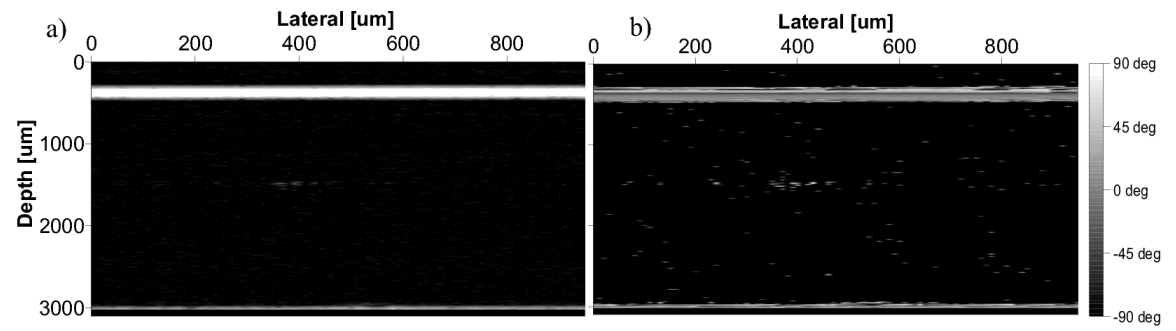

Fig. 4. OCT images of polymer retarder 2: (a) intensity image, (b) optical retardation image.

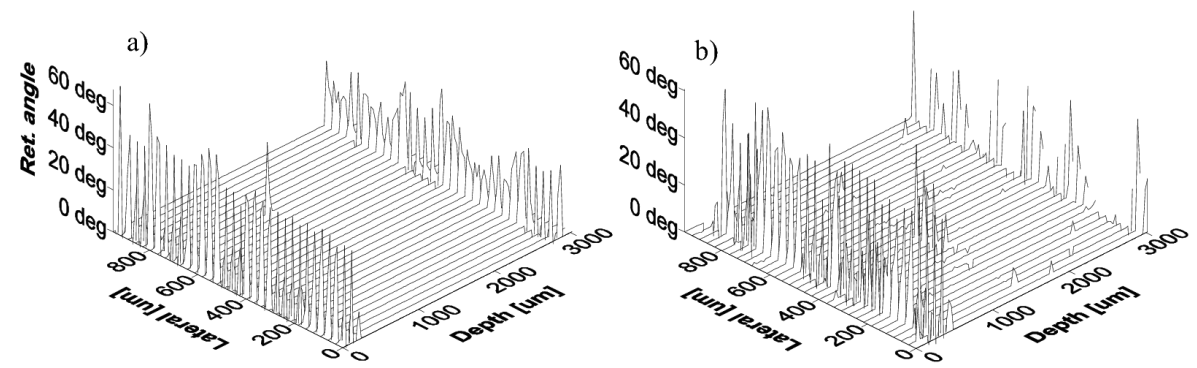

Fig. 5. Wire frame image of optical retardation profile: (a) retarder 1, (b) retarder 2.

images (Fig. 1b and Fig. 2b), one can see that with the use of polarization sensitive analysis it is possible to obtain more sharp OCT images with details which 
TABLE

The PS-OCT system features.

\begin{tabular}{l|c}
\hline \hline \multicolumn{1}{c|}{ Item } & Value \\
\hline lateral scanning resolution & $10 \mu \mathrm{m}$ \\
depth scanning resolution & $4 \mu \mathrm{m}$ \\
lateral scanning velocity & $200 \mu \mathrm{m} / \mathrm{s}$ \\
light source type & photonic fibre source \\
central wavelength & $1550 \mathrm{~nm}$ \\
optical spectral width & $400 \mathrm{~nm}$ \\
emitting power of light & $42 \mathrm{~mW}$
\end{tabular}

are hard to detect by intensity OCT analysis. According to our studies and obtained results, with the aid of polarization diversity detection the visualization contrast is insensitive to polarization state changes of backscattered light, which are introduced by investigated sample. This assumption is true for non-dichroic materials. However, the imaging contrast depends on intensity of backreflected and backscattered light. Moreover, the optical retardation images (Fig. 1b and Fig. 2b) give some additional information about investigated samples like characteristics of local anisotropy changes. The polarization state analysis can be useful for birefringence features determination of the device under test. The retardation profiles, presented as wire frame images (Fig. 5a and Fig. 5b) were taken for two optical retarders, which provide different retardation angle. Those retarders were made of polymer layers placed on surface of glass plate. Analyzing the wire frame images in Fig. 5a and 5b it is possible to make a birefringence characterization of investigated objects. The amplitude of changes between peaks determines the retardation provided by the sample. Those wire frame images are connected to optical retardation images in Fig. 3b and Fig. 4b, which present the retardation changes provided by the retarders as cross-sectional OCT images.

\section{Conclusions}

Optical coherence tomography is a promising measurement technique employing low-coherence interferometry for materials investigation. Authors present their OCT system for measuring polarization properties of investigated samples. Example measurement results are presented which detail internal structure of highly scattering devices like sand-paper, provide information on PLZT ceramics layers, allow retardation angle of polymer retarders to be determined and demonstrate the possibility of performing non-destructive diagnostics of a wide range of materials. Understandably, presented examples do not cover all applications of low-coherence interferometry in the investigation of technical objects. Authors are continuing their research in this field, planning to investigate technical objects 
with OCT systems. The main objective of this research is increasing measurement sensitivity and improving measurement resolution.

\section{Acknowledgments}

The current investigation is a part of a research project No. N515 020 32/1069 supported by the Ministry of Science and Information Technologies Society in 2007-2008.

\section{References}

[1] D.D. Sampson, Proc. SPIE 5502, 25 (2004).

[2] D. Stifter, Appl. Phys. B 88, 337 (2007).

[3] K. Wiesauer, M. Pirchen, E. Gotzinger, C.K. Hitzenberger, R. Oster, D. Stifter, Composites Sci. Technol. 67, 3051 (2007).

[4] D. Stifter, P. Burgholzer, O. Höglinger, E. Götzinger, C.K. Hitzenberger, Appl. Phys. A 76, 947 (2003).

[5] D.C. Adler, J. Stenger, I. Gorczynska, H. Lie, T. Hensick, R. Spronk, S. Wolohojian, N. Khandekar, J.Y. Jiang, S. Barry, A. E. Cable, R. Huber, J.G. Fujimoto, Opt. Express 15, 15972 (2007).

[6] A.F. Fercher, W. Drexler, C.K. Hitzenberg, Rep. Prog. Phys. 66, 239 (2003).

[7] K. Gut, M. Nowak, T. Pustelny, Mol. Quant. Acoust. 28, 101 (2007).

[8] M. Gnyba, M. Kozanecki, P. Wierzba, Mol. Quant. Acoust. 26, 81 (2005).

[9] M. Strąkowski, J. Pluciński, M. Jędrzejewska-Szczerska, R. Hypszer, M. Maciejewski, B.B. Kosmowski, Sensors Actuators A 142, 104 (2008).

[10] M. Strạkowski, M. Jẹdrzejewska-Szczerska, B.B. Kosmowski, J. Pluciński, R. Hypszer, J. Phys. IV (France) 137, 149 (2006).

[11] M. Strakowski, J. Plucinski, A. Loziński, B.B. Kosmowski, Eur. Phys. J. Special Topics 154, 207 (2008). 